\title{
TRANSITION FRONTS FOR INHOMOGENEOUS MONOSTABLE REACTION-DIFFUSION EQUATIONS VIA LINEARIZATION AT ZERO
}

\author{
TIANYU TAO, BEITE ZHU, AND ANDREJ ZLATOŠ
}

\begin{abstract}
We prove existence of transition fronts for a large class of reaction-diffusion equations in one dimension, with inhomogeneous monostable reactions. We construct these as perturbations of corresponding front-like solutions to the linearization of the PDE at $u=0$. While a close relationship of the solutions to the two PDEs has been well known and exploited for KPP reactions (and our method is an extension of such ideas from [15]), to the best of our knowledge this is the first time such an approach has been used in the construction and study of fronts for non-KPP monostable reactions.
\end{abstract}

\section{INTRODUCTION}

We study transition fronts for the one-dimensional reaction-diffusion equation

$$
u_{t}=u_{x x}+f(x, u),
$$

with an inhomogeneous non-negative reaction $f \geq 0$ satisfying $f(x, 0)=f(x, 1)=0$, and with $u \in[0,1]$. Such PDEs model a host of natural processes such as combustion, chemical reactions, population dynamics and others, with $u$ representing (normalized) temperature, concentration of a reactant, or population density.

Both $u \equiv 0$ and $u \equiv 1$ are equilibrium solutions of (1.1) and one is interested in the study of propagation of reaction in space, that is, invasion of the state $u=0$ by the state $u=1$. An important class of solutions modeling the propagation of reaction are transition fronts. A (right-moving) transition front is any entire solution $u: \mathbb{R}^{2} \rightarrow[0,1]$ of (1.1) which satisfies

$$
\lim _{x \rightarrow-\infty} u(t, x)=1 \quad \text { and } \quad \lim _{x \rightarrow+\infty} u(t, x)=0
$$

for each $t \in \mathbb{R}$. In addition, we also require that for any $\varepsilon>0$ there exists $L_{\varepsilon}<\infty$ such that

$$
\sup _{t \in \mathbb{R}} \operatorname{diam}\{x \in \mathbb{R} \mid \varepsilon \leq u(t, x) \leq 1-\varepsilon\} \leq L_{\varepsilon} .
$$

The definition of a left-moving transition front is similar, with the limits in (1.2) exchanged. We will only study right-moving fronts here because the treatment of both cases is identical, up to a reflection in $x$. We note that the above definition is from [2, 5, 11].

We will consider here the case of monostable reactions, for which $u \equiv 1$ is an asymptotically stable solution while $u \equiv 0$ is unstable. We assume that $f$ is Lipschitz,

$$
\begin{gathered}
f(x, 0)=f(x, 1)=0 \quad \text { for } x \in \mathbb{R}, \\
a(x):=f_{u}(x, 0)>0 \\
1
\end{gathered}
$$


exists, and

$$
a(x) g_{0}(u) \leq f(x, u) \leq a(x) g_{1}(u) \quad \text { for }(x, u) \in \mathbb{R} \times[0,1],
$$

where $g_{0}, g_{1} \in C^{1}([0,1])$ satisfy

$$
\begin{array}{cc}
g_{0}(0)=g_{0}(1)=0, \quad g_{0}^{\prime}(0)= & 1, \quad g_{0}(u)>0 \quad \text { and } \quad g_{0}^{\prime}(u) \leq 1 \quad \text { for } u \in(0,1), \\
g_{1}(0)=0, & g_{1}^{\prime}(0)=1, \quad g_{1}^{\prime}(u) \geq 1 \quad \text { for } u \in[0,1], \\
& \int_{0}^{1} \frac{g_{1}(u)-g_{0}(u)}{u^{2}} d u<\infty .
\end{array}
$$

Finally, we let

$$
a_{-}:=\inf _{x \in \mathbb{R}} a(x) \leq \sup _{x \in \mathbb{R}} a(x)=: a_{+} .
$$

When the reaction $f(x, u)=f(u) \geq 0$ is homogeneous, a special case of transition fronts are traveling fronts. These are of the form $u(t, x)=U(x-c t)$, with some front speed $c$ and front profile $U$ such that $\lim _{s \rightarrow-\infty} U(s)=1$ and $\lim _{s \rightarrow \infty} U(s)=0$, and their study goes back to the seminal works of Kolmogorov, Petrovskii, and Piskunov [4], and Fisher [3]. They considered $K P P$ reactions, a special case of monostable reactions with $g_{1}(u)=u$, and found that for each $c \geq c_{0}:=2 \sqrt{f^{\prime}(0)}$ there is a unique traveling front $u(t, x)=U_{c}(x-c t)$. A simple phaseplane analysis shows that this turns out to be the case for general homogeneous monostable reactions, although with a different $c_{0} \geq 2 \sqrt{f^{\prime}(0)}$. In contrast, ignition reactions, satisfying $f(u)=0$ for $u \in[0, \theta] \cup\{1\}$ and $f(u)>0$ for $u \in(\theta, 1)$ (for some ignition temperature $\theta \in(0,1))$, give rise to a single speed $c_{0}>0$ and a single traveling front.

Despite many developments for homogeneous and space-periodic reactions in the almost eight decades since [3,4] (see the reviews [1,14] and references therein), transition fronts in spatially non-periodic media have only been studied relatively recently. The first existence result, for small perturbations of homogeneous bistable reactions (the latter are such that $f(u)<0$ for $u \in(0, \theta)$ and $f(u)>0$ for $u \in(\theta, 1))$, was obtained by Vakulenko and Volpert [13]. Existence results without a hypothesis of closeness to a homogeneous reaction, for ignition reactions of the form $f(x, u)=a(x) g(u)$ for some homogeneous ignition reaction $g$, were proved by Mellet, Roquejoffre, and Sire [7], and by Nolen and Ryzhik [10] (see also [6] for uniqueness and stability results for these reactions). Existence of fronts for general inhomogeneous ignition reactions as well as for some monostable reactions which are in some sense not too far from ignition ones was proved by Zlatoš [16] (uniqueness and stability for the ignition case was also obtained). All these results are based on recovering a front as a locally uniform limit, along a subsequence, of solutions $u_{n}$ of the Cauchy problem with initial data $u_{n}\left(\tau_{n}, x\right) \approx \chi_{(-\infty,-n)}(x)$, where $\tau_{n} \rightarrow-\infty$ are such that $u_{n}(0,0)=\frac{1}{2}$. Existence of a limit $u$ on $\mathbb{R}^{2}$ is guaranteed by parabolic regularity, and the challenge is to show that $u$ is a transition front. We note that even in the monostable case in [15], when one expects multiple transition fronts, existence of only a single transition front was obtained.

A very different approach has been used by Nolen, Roquejoffre, Ryzhik, and Zlatoš [9], and by Zlatoš [15] to prove existence of multiple transition fronts for inhomogeneous KPP reactions. It is well known that when $f$ is KPP, then there is a close relationship between 
the solutions of (1.1) and those of its linearization

$$
v_{t}=v_{x x}+a(x) v
$$

at $u=0$. The reason for this is that all KPP fronts are pulled, with the front speeds determined by the reaction at $u=0$, which is due to the reaction strength $\frac{f(x, u)}{u}$ being largest at $u=0$ for any fixed $x \in \mathbb{R}$. This is in stark contrast with ignition fronts, which are always pushed because they are "driven" by the reaction at intermediate values of $u$.

One can therefore consider the simpler front-like solutions of (1.11), which are of the form

$$
v_{\lambda}(t, x)=e^{\lambda t} \phi_{\lambda}(x) .
$$

Here $\phi_{\lambda}>0$ is a generalized eigenfunction of the operator $\mathcal{L}:=\partial_{x x}+a(x)$, satisfying

$$
\phi_{\lambda}^{\prime \prime}+a(x) \phi_{\lambda}=\lambda \phi_{\lambda}
$$

on $\mathbb{R}$, which exponentially grows to $\infty$ as $x \rightarrow-\infty$ and exponentially decays to 0 as $x \rightarrow \infty$. If we let $\lambda_{0}:=\sup \sigma(\mathcal{L}) \in\left[a_{-}, a_{+}\right]$be the supremum of the spectrum of $\mathcal{L}$, then it is well known that such $\phi_{\lambda}$ exists precisely when $\lambda>\lambda_{0}$, and is unique if we also require $\phi_{\lambda}(0)=1$.

For KPP reactions one can try to use these solutions to find transition fronts for (1.1) with

$$
\lim _{x \rightarrow \infty} \frac{u_{\lambda}(t, x)}{v_{\lambda}(t, x)}=1
$$

for each $t \in \mathbb{R}$, at least for some $\lambda>\lambda_{0}$. This has been achieved in [9] for KPP reactions which are decaying (as $|x| \rightarrow \infty$ ) perturbations of a homogeneous KPP reaction, and for more general KPP reactions in [15]. In both cases one needs $\lambda_{0}<2 a_{-}$(otherwise it is possible that no transition fronts exist [6]) and $\lambda \in\left(\lambda_{0}, 2 a_{-}\right)$.

In the present paper we show that this linearization approach can be extended to general non-KPP monostable reactions. Our method is an extension of the (relatively simple and robust) approach from [15. There it was discovered that while $v_{\lambda}$ is obviously a super-solution of (1.1) when $g_{1}(u)=u$ (i.e., in the KPP case), one can also use $v_{\lambda}$ to find a sub-solution of the form $\tilde{w}_{\lambda}(t, x)=\tilde{h}_{\lambda}\left(v_{\lambda}(t, x)\right)$, for $\lambda \in\left(\lambda_{0}, 2 a_{-}\right)$and an appropriate $g_{0}$-dependent increasing function $\tilde{h}_{\lambda}:[0, \infty) \rightarrow[0,1)$ with

$$
\tilde{h}_{\lambda}(0)=0, \quad \tilde{h}_{\lambda}^{\prime}(0)=1, \quad \lim _{v \rightarrow \infty} \tilde{h}_{\lambda}(v)=1, \quad \tilde{h}_{\lambda}(v) \leq v \quad \text { on }[0, \infty) .
$$

It follows that $\tilde{w}_{\lambda} \leq v_{\lambda}$, and one can then find a transition front $u_{\lambda}$ between the two using parabolic regularity (see below).

While $\tilde{w}_{\lambda}$ remains a sub-solution for all $g_{1}$ from $(1.8), v_{\lambda}$ need not be anymore a supersolution. However, it turns out that one may still be able to construct a super-solution of the form $w_{\lambda}(t, x)=h_{\lambda}\left(v_{\lambda}(t, x)\right)$, for an appropriate increasing $h_{\lambda}:[0, \infty) \rightarrow[0, \infty)$ such that

$$
h_{\lambda}(0)=0, \quad h_{\lambda}^{\prime}(0)=1, \quad h_{\lambda}^{\prime \prime}(v) \geq 0 \quad \text { on } h_{\lambda}^{-1}([0,1]) .
$$

Once again, we then find a transition front $u_{\lambda}$ between $\tilde{w}_{\lambda}$ and $\min \left\{w_{\lambda}, 1\right\}$.

Moreover, a result of Nadin [8] (see also [11]) shows that once some front exists, then also a (time-increasing) critical front exists. The latter is a transition front $u_{C}$ for (1.1) such that 
if $u \not \equiv u_{C}$ is any other transition front and $u(t, x)=u_{C}(t, x)$ for some $(t, x) \in \mathbb{R}^{2}$, then

$$
\left[u_{C}(t, y)-u(t, y)\right](y-x)<0
$$

for all $y \neq x$. That is, a critical front is the (unique up to time translation) "steepest" transition front for (1.1), and is the inhomogeneous version of the minimal speed front for homogeneous reactions. Indeed, if $f$ is homogeneous, then $u_{C}$ is precisely the traveling front with the minimal speed $c_{0}$.

Thus we obtain the following result.

Theorem 1.1. Assume (1.4)-(1.9), let $\nu:=\sup _{u \in(0,1]} \frac{g_{1}(u)}{u} \geq 1$, and let the supremum of the spectrum of $\mathcal{L}:=\partial_{x x}+a(x)$ be $\lambda_{0}:=\sup \sigma(\mathcal{L}) \in\left[a_{-}, a_{+}\right]$. If $\lambda \in\left(\lambda_{0}, 2 a_{-}\right)$satisfies

$$
\lambda \leq 2 a_{-}-\frac{2 \sqrt{\nu-1}}{\sqrt{\nu}+\sqrt{\nu-1}} a_{+},
$$

then (1.1) has a transition front $u_{\lambda}$ with $\left(u_{\lambda}\right)_{t}>0$, satisfying (1.14). In particular, if $\lambda_{0}$ is smaller than the right-hand side of (1.17), then a critical front $u_{C}$, with $\left(u_{C}\right)_{t}>0$, also exists.

Remarks. 1. If $a(x)=f_{u}(x, 0)$ is constant on $\mathbb{R}$, then $\lambda_{0}=a_{-}=a_{+}$, so the right-hand side of (1.17) is always greater than $\lambda_{0}$. Thus a transition front exists for any $g_{0}, g_{1}$ in this case.

2. The front $u_{\lambda}$ does not have a constant speed in general, but when $f$ is stationary ergodic in $x$, then it almost surely has an asymptotic speed $c_{\lambda}>0$ in the sense that if $X(t)$ is the rightmost point such that $u(t, X(t))=\frac{1}{2}$, then

$$
\lim _{|t| \rightarrow \infty} \frac{X(t)}{t}=c_{\lambda}
$$

3. The result also holds with $v_{\lambda}$ replaced by more general solutions of (1.11) of the form $v_{\mu}(t, x) \equiv \int_{\mathbb{R}} v_{\lambda}(t, x) d \mu(\lambda)$, with $\mu$ a finite non-negative non-zero Borel measure supported on a compact subset of $\left(\lambda_{0}, 2 a_{-}-2 \sqrt{\nu-1}(\sqrt{\nu}+\sqrt{\nu-1})^{-1} a_{+}\right]$(or of $\left(\lambda_{0}, 2 a_{-}\right)$if $\nu=1$ ).

4. The result also applies to the more general equation

$$
u_{t}=\left(A(x) u_{x}\right)_{x}+q(x) u_{x}+f(x, u)
$$

with

$$
0<A_{-} \leq A(x) \leq A_{+}<\infty \quad \text { and } \quad|q(x)| \leq q_{+}<\infty
$$

for all $x \in \mathbb{R}$, provided that $q_{+} \leq 2 \sqrt{(a A)_{-}}$with $(a A)_{-}:=\inf _{x \in \mathbb{R}}[a(x) A(x)]$, where

$$
\lambda_{0}:=\sup _{\psi \in H^{1}(\mathbb{R})} \frac{\int_{\mathbb{R}}\left[-A(x) \psi^{\prime}(x)^{2}+q(x) \psi^{\prime}(x) \psi(x)+a(x) \psi(x)^{2}\right] d x}{\int_{\mathbb{R}} \psi(x)^{2} d x} \quad\left(\geq a_{-}\right)
$$

and $2 a_{-}$is replaced in (1.17) by

$$
\lambda_{1}:=\inf _{x \in \mathbb{R}}\left\{a(x)+\sqrt{(a A)_{-}}\left[\sqrt{(a A)_{-}}-|q(x)|\right] A(x)^{-1}\right\} \quad\left(\leq 2 a_{-}\right) .
$$

We indicate the proofs of Remarks 2-4 after the proof of the theorem. 
Our construction of the super-solution $w_{\lambda}$ is of independent interest and extends to more general equations in several dimensions, possibly with time-dependent coefficients. Hence we state it here as a separate result.

Lemma 1.2. Let the function $f(t, x, u) \geq 0$, positive definite matrix $A(t, x)$, and vector field $q(t, x)$ be all Lipschitz, with $(t, x, u) \in\left(t_{0}, t_{1}\right) \times \mathbb{R}^{d} \times[0,1]$ and some $-\infty<t_{0}<t_{1} \leq \infty$. Assume that $a(t, x) \equiv f_{u}(t, x, u)>0$ exists, (1.4) -(1.9) hold for all $(t, x, u) \in\left(t_{0}, t_{1}\right) \times \mathbb{R}^{d} \times$ $[0,1]$, and define $\nu:=\sup _{u \in(0,1]} \frac{g_{1}(u)}{u} \geq 1$. Let $v>0$ be a solution of

$$
v_{t}=\nabla \cdot(A(t, x) \nabla v)+q(t, x) \cdot \nabla v+a(t, x) v
$$

on $\left(t_{0}, t_{1}\right) \times \mathbb{R}^{d}$. If $\nu>1$ and for some $\alpha \leq(\sqrt{\nu}-\sqrt{\nu-1})^{2}$ (or for some $\alpha<1$ if $\left.\nu=1\right)$,

$$
\nabla v(t, x) \cdot A(t, x) \nabla v(t, x) \leq \alpha a(t, x) v(t, x)^{2}
$$

holds for all $(t, x) \in\left(t_{0}, t_{1}\right) \times \mathbb{R}^{d}$, then there exist increasing functions $\tilde{h}$ satisfying (1.15) and $h$ satisfying (1.16) such that $\tilde{w}:=\tilde{h}(v)$ is a sub-solution of

$$
u_{t}=\nabla \cdot(A(t, x) \nabla u)+q(t, x) \cdot \nabla u+f(t, x, u)
$$

on $\left(t_{0}, t_{1}\right) \times \mathbb{R}^{d}$ and $w:=h(v)$ is a super-solution on $\left[\left(t_{0}, t_{1}\right) \times \mathbb{R}^{d}\right] \cap\{(t, x) \mid w(t, x) \leq 1\}$. Therefore, if $u$ solves (1.19) with

$$
\tilde{w}\left(t_{0}, x\right) \leq u\left(t_{0}, x\right) \leq \min \left\{w\left(t_{0}, x\right), 1\right\}
$$

for all $x \in \mathbb{R}^{d}$, then for all $(t, x) \in\left(t_{0}, t_{1}\right) \times \mathbb{R}^{d}$ we have

$$
\tilde{w}(t, x) \leq u(t, x) \leq \min \{w(t, x), 1\} .
$$

Acknowledgements. All authors were supported in part by the NSF grant DMS-1056327. TT and BZ gratefully acknowledge the hospitality of the Department of Mathematics at the University of Wisconsin-Madison during the REU "Analysis and Differential Equations", where this research was performed.

\section{Proof of Theorem 1.1 (using Lemma 1.2)}

Let $\lambda \in\left(\lambda_{0}, 2 a_{-}\right)$and $v=v_{\lambda}$ be from (1.12), with $\phi=\phi_{\lambda}>0$ from (1.13) with $\lim _{x \rightarrow \infty} \phi(x)=0$ and $\phi(0)=1$. It is proved in [15] that the unique such $\phi$ satisfies

$$
\phi^{\prime}(x)^{2} \leq \alpha a(x) \phi(x)^{2}
$$

for $\alpha:=1-\left(2 a_{-}-\lambda\right) a_{+}^{-1}<1$ and all $x \in \mathbb{R}$, as well as

$$
\phi(x) \geq 2 \phi(y)
$$

for some $L<\infty$ and any $y-x \geq L$.

Since $\alpha \leq(\sqrt{\nu}-\sqrt{\nu-1})^{2}$ is by the definition of $\alpha$ equivalent to

$$
\lambda \leq 2 a_{-}-\left[1-(\sqrt{\nu}-\sqrt{\nu-1})^{2}\right] a_{+}=2 a_{-}-\frac{2 \sqrt{\nu-1}}{\sqrt{\nu}+\sqrt{\nu-1}} a_{+}
$$

(which is (1.17)), Lemma 1.2 applies to $v$ and (1.1). Thus we have (1.21) and a standard limiting argument now recovers an entire solution to (1.1) between $\tilde{w}$ and $\min \{w, 1\}$. We 
let $u_{n}$ be the solution of (1.1) on $(-n, \infty) \times \mathbb{R}$ with $u_{n}(-n, x):=\tilde{w}(-n, x)$. Since $\tilde{w}(t, x) \leq$ $\min \{w(t, x), 1\}$ because $h(v) \geq v$ for $v \in h^{-1}([0,1])$, (1.20) is satisfied with $t_{0}:=-n$ and we have (1.21) on $(-n, \infty) \times \mathbb{R}$. By parabolic regularity, there is a subsequence of $\left\{u_{n}\right\}$ which converges, locally uniformly on $\mathbb{R}^{2}$, to an entire solution $u$ of (1.1). We obviously have

$$
\tilde{w} \leq u \leq \min \{w, 1\}
$$

and (1.14) for $u_{\lambda}:=u$ follows from $\tilde{h}^{\prime}(0)=h^{\prime}(0)=1$. We also have $u_{t} \geq 0$, since the same is true for $u_{n}$ due to $\tilde{w}_{t}=h^{\prime}(v) v_{t} \geq 0$ and the maximum principle for $\left(u_{n}\right)_{t}$ (which satisfies a linear equation and is non-negative at $t=-n)$. The strong maximum principle then gives $u_{t}>0$ because obviously $u_{t} \not \equiv 0$. Finally, $u$ is a transition front because the second limit in (1.2) follows from $\lim _{x \rightarrow \infty} \phi(x)=0$ and (1.16), and (1.3) holds with

$$
L_{\varepsilon}:=L\left\lceil\log _{2}\left(\tilde{h}^{-1}(1-\varepsilon)-h^{-1}(\varepsilon)\right)\right\rceil
$$

due to (2.3) and (2.2) (with $\tilde{h}, h$ from the lemma). The first limit in (1.2) is then obvious from $u \leq 1$, and the proof is finished by using the abovementioned result from [8] for critical fronts.

The claim in Remark 2 is proved as an analogous statement in [15, Theorem 1.2].

The claim in Remark 3 holds because $L$ can be chosen uniformly for all $\lambda$ in the support of $\mu$ [15] and so (2.2) holds with $\phi(\cdot)$ replaced by $v_{\mu}(t, \cdot)$. Also, $v_{\mu}$ satisfies (2.1) with $\alpha$ corresponding to $\lambda:=\sup \operatorname{supp} \mu$.

The claim in Remark 4 holds because (2.1) and (2.2) continue to hold in that case, albeit with $2 a_{-}$replaced by $\lambda_{1}$ in the definition of $\alpha[15]$.

\section{Proof of Lemma 1.2}

[15] shows that there is an increasing $\tilde{h}=\tilde{h}_{\lambda}$ as in (1.15) such that $\tilde{w}(t, x):=\tilde{h}(v)$ is a sub-solution of (1.1). This yields the first inequality in (1.21). We will next find an increasing $h=h_{\lambda}$ as in (1.16) such that $w(t, x):=h(v(t, x))$ will be a super-solution where $w(t, x) \leq 1$, which will yield the second inequality because $u \leq 1$ by the hypotheses. Our proof will be a super-solution counterpart to the sub-solution argument in [15]; it was a little surprising to us that such a counterpart argument exists for non-KPP reactions.

If $h$ is as in (1.16), then (1.18) shows that

$$
\begin{aligned}
w_{t}-\nabla \cdot(A \nabla w)-q \cdot \nabla w & =h^{\prime}(v)\left[v_{t}-\nabla \cdot(A \nabla v)-q \cdot \nabla v\right]-h^{\prime \prime}(v) \nabla v \cdot B \nabla v \\
& =h^{\prime}(v) a v-h^{\prime \prime}(v) \nabla v \cdot B \nabla v \\
& \geq a\left[v h^{\prime}(v)-\alpha v^{2} h^{\prime \prime}(v)\right]
\end{aligned}
$$

when $w(t, x) \leq 1$. We can then conclude that $w$ is a super-solution of (1.1) where $w(t, x) \leq 1$ once we show that on $h^{-1}([0,1])$ we also have

$$
v h^{\prime}(v)-\alpha v^{2} h^{\prime \prime}(v) \geq g_{1}(h(v)) .
$$

It therefore remains to find $h$ satisfying (1.16) and (3.1). We let $c:=\alpha^{1 / 2}+\alpha^{-1 / 2}$ and notice that since $\gamma+\gamma^{-1} \geq 2 \sqrt{\nu}$ for all positive $\gamma \leq \sqrt{\nu}-\sqrt{\nu-1}$, the hypothesis $\alpha \leq(\sqrt{\nu}-\sqrt{\nu-1})^{2}$ 
yields $c \geq 2 \sqrt{\nu}$. Next let $U$ be the unique solution to the ODE

$$
U^{\prime \prime}+c U^{\prime}+g_{1}(U)=0
$$

on $\left[s_{0}, \infty\right)$, with

$$
U\left(s_{0}\right)=1 \quad \text { and } \quad U^{\prime}\left(s_{0}\right)=-\sqrt{\alpha} g_{1}(1),
$$

where $s_{0} \in \mathbb{R}$ will be chosen later. (This is the ODE that would be satisfied by the traveling front profile with speed $c$ for the homogeneous reaction $g_{1}(u)$ if we had $g_{1}(1)=0$; this profile would then also satisfy $\lim _{s \rightarrow-\infty} U(s)=1$ and $\lim _{s \rightarrow-\infty} U^{\prime}(s)=0$ instead of (3.3).)

Notice that $U^{\prime}\left(s_{0}\right) \geq-\frac{c}{2}$ because $g_{1}(1) \leq \nu$ and

$$
\sqrt{\alpha} \leq(\sqrt{\nu}+\sqrt{\nu-1})^{-1} \leq \nu^{-1 / 2} .
$$

Let $V(s):=U^{\prime}(s)$, and consider the curve $\theta:=\{(U(s), V(s))\}_{s \geq s_{0}}$. It is easy to see that $\theta$ cannot leave the closed triangle $T$ in the $(U, V)$ plane with sides $V=0, U=1$, and $V=-\frac{c}{2} U$. This is because $\left(U\left(s_{0}\right), V\left(s_{0}\right)\right) \in T$ and on $\partial T$, the vector field $\left(V,-c V-g_{1}(U)\right)$ either points inside $T$ or is parallel to $\partial T$. Here we use $c \geq 2 \sqrt{\nu}$ to obtain on the third side

$$
\left(\frac{c}{2}, 1\right) \cdot\left(-\frac{c}{2} U,-c\left(-\frac{c}{2} U\right)-g_{1}(U)\right)=\frac{c^{2}}{4} U-g_{1}(U) \geq \nu U-g_{1}(U) \geq 0 .
$$

It follows that $U^{\prime}(s)<0$ on $\left[s_{0}, \infty\right)$, and since $g_{1}(U(s))>0, U(s)$ cannot have local minima on $\left[s_{0}, \infty\right)$. Hence $\lim _{s \rightarrow \infty} U(s)$ exists and $\lim _{s \rightarrow \infty} U^{\prime}(s)=0$. Finally, $g_{1}>0$ on $(0,1]$ yields $\lim _{s \rightarrow \infty} U(s)=0$.

We now define $h(0):=0$ and

$$
h(v):=U\left(-\alpha^{-1 / 2} \ln v\right)
$$

for $v \in\left(0, e^{-\sqrt{\alpha} s_{0}}\right]$, so $h$ is increasing and continuous at 0 , with $h\left(e^{-\sqrt{\alpha} s_{0}}\right)=1$ (we then extend $h$ onto $[0, \infty)$ arbitrarily, only requiring that it be increasing). Since $c>2 \sqrt{g_{1}^{\prime}(0)}=2$ and

$$
\int_{0}^{1} \frac{g_{1}(u)-u}{u^{2}} d u<\infty
$$

by (1.7) and (1.9), a result of Uchiyama [12, Lemma 2.1] $\operatorname{shows} \lim _{s \rightarrow \infty} U(s) e^{\sqrt{\alpha} s} \in(0, \infty)$. (This result assumes $g_{1}(1)=0$ but we can extend $g_{1}, U$ to $[0,2]$ so that $g_{1}(2)=0$ and $U$ satisfies (3.2), and then apply [12] to $\tilde{g}(u):=\frac{1}{2} g_{1}(2 u)$ and the function $\tilde{U}(s):=\frac{1}{2} U(s)$.)

If we now pick the unique $s_{0}$ in (3.3) such that $\lim _{s \rightarrow \infty} U(s) e^{\sqrt{\alpha} s}=1$ (notice that (3.2) is an autonomuous ODE), we obtain $h^{\prime}(0)=1$. We also have (3.1) on $\left[0, e^{-\sqrt{\alpha} s_{0}}\right]$ because on that interval, (3.2) immediately yields

$$
\alpha v^{2} h^{\prime \prime}(v)-v h^{\prime}(v)+g_{1}(h(v))=0 .
$$

It therefore remains to show that $h^{\prime \prime}(v) \geq 0$ on $\left[0, e^{-\sqrt{\alpha} s_{0}}\right]$. Due to (3.6) and (3.5), this is equivalent to

$$
-U^{\prime}(s) \geq \sqrt{\alpha} g_{1}(U(s))
$$

for $s \geq s_{0}$. Thus we need to show that $\theta$ stays at or below $\psi:=\left\{\left(U(s),-\sqrt{\alpha} g_{1}(U(s))\right)\right\}_{s \geq s_{0}}$. This is true at $s=s_{0}$ by the definition of $U$, so it is sufficient to show that on $\psi$, the vector field $\left(V,-c V-g_{1}(U)\right)$ points either below or parallel to $\psi$. This holds because the normal 
vector to $\psi$ pointing down is $\left(\sqrt{\alpha} g_{1}^{\prime}(U) V, V\right)$, so on $\psi$ we have

$$
\begin{aligned}
\left(V,-c V-g_{1}(U)\right) \cdot\left(\sqrt{\alpha} g_{1}^{\prime}(U) V, V\right) & =\alpha^{1 / 2} g_{1}^{\prime}(U) V^{2}-\left(\alpha^{1 / 2}+\alpha^{-1 / 2}\right) V^{2}-\alpha^{1 / 2} g_{1}(U) \alpha^{-1 / 2} V \\
& =\alpha^{1 / 2}\left(g_{1}^{\prime}(U)-1\right) V^{2},
\end{aligned}
$$

which is non-negative due to (1.8) and $U(s) \leq 1$ for $s \geq s_{0}$. It follows that $h^{\prime \prime}(v) \geq 0$ on $\left[0, e^{-\sqrt{\alpha} s_{0}}\right]$ and so $h$ satisfies (1.16) and (3.1). The proof is finished.

\section{REFERENCES}

[1] H. Berestycki, The influence of advection on the propagation of fronts in reaction-diffusion equations, Nonlinear PDEs in Condensed Matter and Reactive Flows, NATO Science Series C, 569, H. Berestycki and Y. Pomeau eds, Kluwer, Doordrecht, 2003.

[2] H. Berestycki and F. Hamel, Generalized transition waves and their properties, Comm. Pure Appl. Math 65 (2012), 592-648.

[3] R. Fisher, The wave of advance of advantageous genes, Ann. Eugenics 7 (1937), 355-369.

[4] A.N. Kolmogorov, I.G. Petrovskii and N.S. Piskunov, Étude de l'équation de la chaleur de matière et son application à un problème biologique, Bull. Moskov. Gos. Univ. Mat. Mekh. 1 (1937), 1-25.

[5] H. Matano, talks at several conferences.

[6] A. Mellet, J. Nolen, J.-M. Roquejoffre and L. Ryzhik, Stability of generalized transition fronts, Commun. PDE 34 (2009), 521-552.

[7] A. Mellet, J.-M. Roquejoffre and Y. Sire, Generalized fronts for one-dimensional reaction-diffusion equations, Discrete Contin. Dyn. Syst. 26 (2010), 303-312.

[8] G. Nadin, Critical travelling waves for general heterogeneous one dimensional reaction-diffusion equation, preprint.

[9] J. Nolen, J.-M. Roquejoffre, L. Ryzhik, and A. Zlatoš, Existence and non-existence of Fisher-KPP transition fronts, Arch. Ration. Mech. Anal. 203 (2012), 217-246.

[10] J. Nolen and L. Ryzhik, Traveling waves in a one-dimensional heterogeneous medium, Ann. Inst. H. Poincaré Anal. Non Linéaire 26 (2009), 1021-1047.

[11] W. Shen, Traveling waves in diffusive random media, J. Dynam. Differ. Equat. 16 (2004), 1011-1060.

[12] K. Uchiyama, The behavior of solutions of some non-linear diffusion equations for large time, J. Math. Kyoto Univ. 18 (1978), 453-508.

[13] S. Vakulenko and V. Volpert, Generalized travelling waves for perturbed monotone reaction-diffusion systems, Nonlinear Anal. 46 (2001), 757-776.

[14] J. Xin, Front propagation in heterogeneous media, SIAM Rev. 42 (2000), 161-230.

[15] A. Zlatoš, Transition fronts in inhomogeneous Fisher-KPP reaction-diffusion equations, J. Math. Pures Appl. 98 (2012), 89-102.

[16] A. Zlatoš, Generalized traveling waves in disordered media: Existence, uniqueness, and stability, Arch. Ration. Mech. Anal. 208 (2013), 447-480.

University of Wisconsin, Madison, WI 53706, USA, EmaIL: ttao@wisc.edu

University of California, Berkeley, CA 94720, USA, Email: jupiter_ju@berkeley.edu

Department of Mathematics, University of Wisconsin, Madison, Wi 53706, USA

EMAIL: zlatos@math.wisc.edu 